\title{
Evaluation of cardiac function in patients with liver cirrhosis using tissue Doppler study
}

\author{
Mohammed M. Ashmawy ${ }^{a}$, Hosny A. Younis ${ }^{b}$, Mahmoud A. Abd Elbaset ${ }^{c}$, \\ Haitham A. Abdel Rahman ${ }^{b}$, Ahmed M. Ashmawy ${ }^{a}$, Muhammad A. Shawky ${ }^{d}$, \\ Mohammed M. Abd Elnasser ${ }^{\mathrm{b}}$
}

\author{
${ }^{a}$ Department of Internal Medicine, Assuit \\ University, Departments of ${ }^{\mathrm{b}}$ Internal Medicine, \\ ${ }^{\mathrm{c} C a r d i o v a s c u l a r}$ Diseases, ${ }^{\mathrm{d}}$ Tropical Medicine \\ and Gastroenterology, AL-Azhar University, \\ Assuit, Egypt \\ Correspondence to Muhammad Abd El-Gawad \\ Shawky, MD, Department of Tropical Medicine \\ and Gastroenterology, Al-Azhar University, \\ Assiut, 71524, Egypt. Tel: +20 884763 588; \\ fax: 0884763588; \\ e-mail: mgawwad2013@gmail.com
}

Received 25 April 2018

Accepted 9 May 2018

The Egyptian Journal of Internal Medicine 2018, 30:115-120

\begin{abstract}
Background and aim
Cardiac dysfunction is a prevalent finding in patients with liver cirrhosis. We aimed to evaluate left ventricular function by tissue Doppler imaging in patients with liver cirrhosis.
\end{abstract}

\section{Patients and methods}

This is a cross-sectional case-control study that involved 90 patients with liver cirrhosis: 30 patients with Child $A, 30$ patients with Child $B$, and 30 patients with Child $\mathrm{C}$ cirrhosis. Moreover, 45 healthy volunteers were included in the study as a control group. All patients and controls were examined by conventional Doppler and tissue Doppler echocardiography.

\section{Results}

Patients with liver cirrhosis showed significantly lower ejection fraction (EF) levels than control group $(P=0.001)$, but only nine patients showed EF levels less than 55 . Moreover, there is significantly decrease in EF in patients with decompensated cirrhosis than those with compensated cirrhosis $(P=0.005)$. A total of 60 patients showed diastolic dysfunction: 10 patients with Child $A, 20$ patients with Child $B$, and 30 patients with Child $\mathrm{C}$ cirrhosis. There were significantly differences between patients with liver cirrhosis and control group in the other parameters of systolic (S wave and myocardia performance index) and diastolic (early and late velocity and deceleration and isovolumetric relaxation time) functions.

\section{Conclusion}

Patients with liver cirrhosis showed significantly decreased left ventricular systolic and diastolic functions than control group, which is more pronounced in decompensated than compensated patients.

\section{Keywords:}

Keywords, cirrhotic cardiomyopathy, diastolic dysfunction, echocardiography, left ventricular function, liver cirrhosis, tissue Doppler imaging

Egypt J Intern Med 30:115-120

(C) 2018 The Egyptian Journal of Internal Medicine $1110-7782$

\section{Introduction}

Cardiomyopathy in patients with cirrhosis is defined as 'chronic cardiac dysfunction in patients with cirrhosis characterized by impaired contractile responsiveness to stress and/or altered diastolic relaxation with electrophysiological abnormalities in the absence of other known cardiac disease' [1-3]. Diagnosis of cardiac dysfunction in patients with cirrhosis mostly depends on noninvasive modalities. The importance of cardiac imaging has been increased, and the appearance of newer imaging modalities is associated with increased diagnostic and prognostic aspects of cirrhotic cardiac dysfunction $[4,5]$.

\section{Systolic dysfunction}

The most commonly used parameter to assess left ventricular (LV) function is the ejection fraction (EF) which is mostly assessed by echocardiography and estimated from the end systolic and end diastolic volume of the LV [6,7]. EF is usually calculated by the modified Simpson's rule [8]. Two-dimensional echocardiography is usually inaccurate, and the threedimensional echocardiography which has accuracy and reproducibility comparable to cardiac MRI should be used instead of it [9].

Tissue Doppler imaging (TDI) echocardiography is a new modality that detects early deformity and alteration of the myocardial fibers in patients with cirrhosis even in the absence of volume overload and before affecting the function of the heart [10]. Patients with liver cirrhosis showed significantly reduced $\mathrm{S}$ wave

This is an open access journal, and articles are distributed under the term of the Creative Commons Attribution-NonCommercial-ShareAlike 4.0 License, which allows others to remix, tweak, and build upon the work non-commercially, as long as appropriate credit is given and the new creations are licensed under the identical terms. 
value during TDI examination when compared with healthy controls [4].

\section{Diastolic dysfunction}

Decreased early diastolic filling of the LV with subsequent increase in atrial filling is the most frequently encountered diastolic dysfunction in patients with cirrhosis (ratio $<1$ ), increased resistance to blood flow to $\mathrm{LV}$ results in prolonged isovolumetric relaxation time (IVRT), and prolonged deceleration time (DT)

is also frequently found in patients with cirrhosis [4]. A key factor for diagnosis of diastolic dysfunction is the estimation of decreased $\mathrm{E}^{\prime}$ velocity at the mitral valve annulus by TDI. Filling pressure during diastole can be estimated by the ratio of $\mathrm{E}$ wave (mitral inflow by TDI) to $\mathrm{E}^{\prime}$ wave (E/E' ratio) [11].

\section{Patients and methods}

Our study is a cross-sectional case-control study conducted at Al-Azhar Assiut University Hospital, Department of Internal Medicine. The study included 90 patients with liver cirrhosis and 45 healthy volunteers cross-matched with the patients in age and sex. The study populations were divided into four groups:

(1) Group 1 included 30 patients with Child A liver cirrhosis.

(2) Group 2 included 30 patients with Child B liver cirrhosis.

(3) Group 3 included 30 patients with Child C liver cirrhosis.

(4) Control group included 45 healthy volunteers.

\section{Inclusion criteria}

The study included any adult patient with liver cirrhosis above the age of 18 years.

\section{Exclusion criteria}

Absence of liver cirrhosis; patients with hyperdynamic circulation such as those with anemia or pregnancy; patients with cardiac diseases, either congenital or acquired; patients with hypertension or diabetes mellitus; patients with renal or pulmonary diseases; patients taking drugs affecting cardiac functions such as $\beta$-blockers or positive or negative inotropic drugs; and patients with recent hemorrhage were excluded.

The patients and controls were subjected to the following: full history taking, full clinical examination, laboratory studies, chest radiography, and abdominal ultrasound for confirmation of liver cirrhosis. A 12-lead resting EGG examination was done for each one of the study population in the supine position immediately before echocardiographic examination. An informed consent was obtained from each one of the participating persons.

\section{Echocardiographic examination}

Echocardiography was done by the same operator for all of the participants. Participants were examined at rest in the left lateral decubitus position using A vivid 7, General Electric, Milwakee, USA with a $2-4 \mathrm{MHz}$ transducer with the following modalities of echo: $2 \mathrm{D}$ echo, $\mathrm{M}$ mode echo, color Doppler echo, CW and PW Doppler, and pulsed TDI. Complete transthoracic echocardiographic examination including conventional echocardiography and tissue Doppler echocardiography was done according to the American Society Guidelines of Echocardiography [12]. Echocardiographic examinations were performed after $20-30 \mathrm{~min}$ of rest with the patient in quiet respiration in the partial left lateral decubitus position, and accompanied by recording resting ECG. The following views were used:

(1) Parasternal long-axis view.

(2) Parasternal short-axis view.

(3) Apical four-chamber and two-chamber views.

(4) Subcostal view in some patients with poor echo window.

\section{Assessment of left ventricular function}

LV function was assessed by the following:

(1) EF calculation was done using the following:

(a) M mode method: measuring the dimension of $\mathrm{LV}$, from the leading edge of septal endocardial echo to the leading edge of posterior wall of endocardium using Tiecheol's equation:

Ejection fraction $(\%)=\left(\frac{\operatorname{LVIDd}^{3} \mathrm{LVISd}^{3}}{\mathrm{~L}}\right)$.

(b) Biplane method: done by manual tracing of the endocardial border of LV in the apical four-chamber and apical two-chamber views for detecting LV end diastolic volume and LV end systolic volume in both views for calculating $\mathrm{EF}$.

(2) Pulsed-wave myocardial performance index (PWMPI): mitral inflow and LV outflow velocity-time intervals were used to measure Doppler time intervals including the following:

(a) The interval 'A' from the cessation to the onset of mitral inflow was equal to the sum of isovolumetric contraction time (IVCT), ejection time (ET), and IVRT. 
(b) LV ET 'B' was the duration of the LV ejection during systole. Thus, the sum of IVCT and IVRT was obtained by subtracting ' $\mathrm{B}$ ' from 'A'.

The MPI was calculated as (A-B)/B.

\section{Tissue Doppler imaging}

By activating the TDI function in the echocardiography machine, the mitral annular velocities were recorded using the pulsed-wave DTI. A variable frequency phased array transducer $(2.0-4.0 \mathrm{MHz})$ was used. The filter settings were kept low $(50 \mathrm{~Hz})$, and gains were adjusted at the optimal level for good-quality velocity.

From the apical four-chamber view, the following was calculated: the longitudinal mitral annular velocities were recorded from septal, lateral, anterior, and inferior LV sites, which may show. (a) the positive peak systolic velocity when the mitral ring moved toward the cardiac apex owing to the longitudinal contraction of the LV ( $\mathrm{S}^{\prime}$ wave) and (b) two negative diastolic velocities when the mitral annulus moved toward the base away from the apex, one during the early, anterior and inferior LV sites. A mean value of the aforementioned four sites was used to assess global systolic function.

\section{Myocardial performance index by TDI}

This was done by TDI velocity-time intervals which measured from the mitral annulus at the septal and lateral segments. (a) TDI IVCT was measured between cessation of $\mathrm{A}^{\prime}$ wave and onset of $\mathrm{S}^{\prime}$ wave; (b) TDI ET was obtained between onset and cessation of $\mathrm{S}^{\prime}$ wave; (c) TDI IVRT was obtained between cessation of $\mathrm{S}$ wave and onset of $\mathrm{E}^{\prime}$ wave; and (d) MPI TDI was calculated as $(\mathrm{ICT}+\mathrm{IVRT}) /(\mathrm{ET})$.

\section{Average $S$ wave}

The mitral annular positive peak systolic velocities were recorded from septal, lateral, anterior, and inferior LV sites. A mean value for the aforementioned four sites was used to assess global systolic function.

\section{Assessment of left ventricular diastolic function}

The assessment of left ventricular diastolic function was done using the Doppler beam aligned to the direction of flow, and a $1-2 \mathrm{~mm}$ sample volume placed between the tips of the mitral leaflets during diastole, in the apical four-chamber view, for detecting the following:

(1) Transmitral early velocity wave (E wave).

(2) Transmitral late velocity wave (A wave).

(3) $\mathrm{E} / \mathrm{A}$ ratio (normally $>0.8$ ).
(4) DT measured along the descending slope of mitral flow A wave.

(5) IVRT measured by TDI-PWD at LV basal lateral wall from the end of systolic velocity wave (S wave) to the onset of early diastolic wave (É wave).

(6) $\mathrm{MV} \mathrm{E} / \mathrm{E}^{\prime}$ ratio measured by TDI-PWD to obtain mitral inflow.

\section{Ethical consideration}

The study is approved by the ethical committee of Faculty of Medicine, Al-Azhar University, Assiut. An informed consent was obtained from each patient.

\section{Statistical analysis}

The data were tested for normality using the Anderson-Darling test and for homogeneity variances before further statistical analysis. Categorical variables were described by number and percentage, whereas continuous variables were described by mean and SD. $\chi^{2}$-test and Fisher's exact test were used to compare between categorical variables, whereas comparison between continuous variables was done by $t$-test and analysis of variance. A two-tailed $P$ value of less than 0.05 was considered statistically significant. All analyses were performed with the IBM SPSS 22 software (IBM SPSS Inc., Chicago, US) for windows 10.

\section{Results}

Our study included 90 patients with liver cirrhosis, with 56 males and 34 females, with age ranging from 39 to 63 years. In addition, 45 healthy volunteers were cross-matched in age and sex with patients. Laboratory data in patients and controls are showed in Table 1, whereas Table 2 showed comparison between patient groups and controls in laboratory data.

\section{Echocardiographic characteristics}

Nine patients in our study showed EF levels less than 55 , and 40 patients were diagnosed with diastolic dysfunction depending on E/A ration below 1, DT higher than $200 \mathrm{~ms}$, and IVRT more than $100 \mathrm{~ms}$ [2].

Table 1 Comparison between patients and controls in laboratory data

\begin{tabular}{lccc}
\hline Factors & Patients & Control & $P$ value \\
\hline AST $(\mu \mathrm{l} / \mathrm{l})$ & $67.02 \pm 22.39$ & $30.46 \pm 6.5$ & 0.00 \\
ALT $(\mu \mathrm{l} / \mathrm{l})$ & $87.87 \pm 31.29$ & $27.77 \pm 6.6$ & 0.00 \\
PT $(\mathrm{s})$ & $17.61 \pm 2.87$ & $12.04 \pm 0.64$ & 0.00 \\
Creatinine $(\mathrm{mg} / \mathrm{dl})$ & $0.96 \pm 0.24$ & $0.90 \pm 0.20$ & 0.105 \\
Albumin $(\mathrm{g} / \mathrm{dl})$ & $3.01 \pm 0.73$ & $4.03 \pm 0.28$ & 0.00 \\
Hb $(\mathrm{g} / \mathrm{dl})$ & $12.44 \pm 1.09$ & $13.56 \pm 1.16$ & 0.00 \\
Bilirubin $(\mathrm{mg} / \mathrm{dl})$ & $3.66 \pm 2.79$ & $0.86 \pm 0.17$ & 0.00 \\
\hline
\end{tabular}

ALT, alanine aminotransferase; AST, aspartate aminotransferase; $\mathrm{Hb}$, hemoglobin; $\mathrm{PT}$, prothrombin time; RBS, random blood sugar. 
Patients with liver cirrhosis showed significantly decreased both LV systolic and diastolic function than control group. These LV dysfunctions increased by the increase in the severity of liver disease presented in Child score. Patients with compensated liver cirrhosis have less impaired LV function than those with decompensated cirrhosis (Tables 3-5).

\section{Discussion}

Depending on the Simpson and M mode methods in estimation of $\mathrm{EF}$, nine ((10\%) patients showed $\mathrm{EF}$ levels less than 55. Diastolic dysfunction, as defined in the 2005 World Congress of Gastroenterology (E/A ratio $<1.0, \mathrm{DT}>200 \mathrm{~ms}$, and IVRT $>80 \mathrm{~ms}$ ), is highly prevalent in patients with cirrhosis [2]. Depending on these criteria, $60(66.6 \%)$ patients in our study were diagnosed as having diastolic dysfunction.

Our study agrees with Hammami et al. [13] who compared 80 patients with cirrhosis with 80 healthy participants and found that 14 (17.5\%) patients had EF less than 55 and 49 (61.2\%) patients had diastolic dysfunction. Karagiannakis et al. [14] studied diastolic

Table 2 Comparison between the study groups regarding laboratory data

\begin{tabular}{lcccc}
\hline Factors & Group A & Group B & Group C & Controls \\
\hline AST $(\mu \mathrm{l} / \mathrm{l})$ & $50.33 \pm 17.34^{*}$ & $23.74 \pm 4.33^{\dagger}$ & $15.05 \pm 2.74^{\dagger}$ & $30.46 \pm 6.5^{\ddagger}$ \\
ALT $(\mu \mathrm{l} / \mathrm{l})$ & $80.13 \pm 29.98^{*}$ & $99.86 \pm 39.93^{\dagger}$ & $83.63 \pm 16.77^{\star}$ & $27.77 \pm 6.6^{\ddagger}$ \\
PT (s) & $14.72 \pm 0.89^{\star}$ & $17.24 \pm 1.14^{\dagger}$ & $20.86 \pm 1.85^{\ddagger}$ & $12.04 \pm 0.64^{\S}$ \\
Creatinine (mg/dl) & $0.86 \pm 0.26^{\star}$ & $1 \pm 0.23^{\dagger}$ & $1.03 \pm 0.19^{\dagger}$ & $0.90 \pm 0.20^{\star}$ \\
Albumin (g/dl) & $3.81 \pm 0.30^{\star}$ & $3.11 \pm 0.20^{\dagger}$ & $2.16 \pm 0.32^{\dagger}$ & $4.03 \pm 0.28^{\S}$ \\
Hb (g/dl) & $13.41 \pm 0.95^{\star}$ & $11.93 \pm 0.76^{\dagger}$ & $11.98 \pm 0.82^{\dagger}$ & $13.56 \pm 1.16^{\star}$ \\
Bilirubin (mg/dl) & $1.7 \pm 0.24^{\star}$ & $2.47 \pm 0.35^{\dagger}$ & $6.8 \pm 2.87^{\ddagger}$ & $0.86 \pm 0.17^{\S}$ \\
RBS (mg/dl) & $128.13 \pm 12.42^{\star}$ & $115.46 \pm 16.18^{\dagger}$ & $110.3 \pm 18.45^{\dagger}$ & $113.95 \pm 14.33^{\dagger}$ \\
\hline
\end{tabular}

Between groups with different symbols, but no significant differences between groups with the same symbol. ALT, alanine

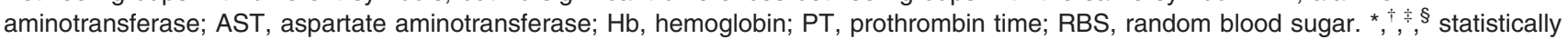
significant differences.

Table 3 Comparison between patients and controls in echocardiographic findings

\begin{tabular}{|c|c|c|c|}
\hline & Cases $(N=90)$ & Control $(N=45)$ & $P$. value \\
\hline MPI PWD & $0.43 \pm 0.04$ & $0.4 \pm 0.02$ & $<0.001$ \\
\hline MPI TDI & $0.5 \pm 0.08$ & $0.47 \pm 0.03$ & $<0.001$ \\
\hline EF by Simpson & $61.38 \pm 7.3$ & $65.18 \pm 4.2$ & $<0.001$ \\
\hline Average S wave & $11.32 \pm 2.16$ & $12.6 \pm 1.27$ & $<0.001$ \\
\hline EF By M Mode & $61.43 \pm 7.52$ & $65.71 \pm 3.92$ & $<0.001$ \\
\hline MV E/É ratio & $14.97 \pm 3.06$ & $5.31 \pm 1.03$ & $<0.001$ \\
\hline MV E/A ratio & $0.75 \pm 0.2$ & $1.35 \pm 0.12$ & $<0.001$ \\
\hline DT (ms) & $234.8 \pm 18.9$ & $193.18 \pm 12.3$ & $<0.001$ \\
\hline IVRT (ms) & $112.07 \pm 13.52$ & $75.27 \pm 5.8$ & $<0.001$ \\
\hline
\end{tabular}

A, late velocity wave; DT, deceleration time; $\mathrm{E}$, early velocity wave; $\mathrm{E}^{\prime}$, early diastolic wave; EF, ejection fraction; IVRT, isovolumetric relaxation time; MPI, myocardial performance index; MV, mitral valve; PWD, pulsed-wave Doppler; S, systolic velocity wave; TDI, tissue Doppler imaging. $P<0.05$ considered significant.

Table 4 Comparison between the study groups in echocardiographic findings

\begin{tabular}{|c|c|c|c|c|}
\hline Factors & Group 1 & Group 2 & Group 3 & Controls \\
\hline MPI PWD & $0.41 \pm 0.021^{*}$ & $0.43 \pm 0.037^{\dagger}$ & $0.44 \pm 0.056^{\dagger}$ & $0.40 \pm 0.022^{*}$ \\
\hline MPI TDI & $0.46 \pm 0.026^{\star}$ & $0.50 \pm 0.069^{\dagger}$ & $0.53 \pm 0.10^{\dagger}$ & $0.46 \pm 0.025^{\star}$ \\
\hline EF Simpson & $63.93 \pm 4.6^{\star}$ & $61.53 \pm 6.7^{*}$ & $58.66 \pm 9.03^{\dagger}$ & $65.17 \pm 4.20^{\star}$ \\
\hline Average S wave & $12.33 \pm 1.34^{*}$ & $11.58 \pm 2.16^{\star}$ & $10.05 \pm 2.23^{\dagger}$ & $12.60 \pm 1.26^{*}$ \\
\hline EF M mode & $64.3 \pm 3.91^{*}$ & $61.56 \pm 6.98^{*}$ & $58.43 \pm 9.57^{\dagger}$ & $65.71 \pm 3.91^{*}$ \\
\hline MV E/E' & $13.33 \pm 4.45^{\star}$ & $15.40 \pm 1.84^{\dagger}$ & $16.16 \pm 1.01^{\dagger}$ & $5.31 \pm 1.01^{\ddagger}$ \\
\hline MV E/A & $0.88 \pm 0.28^{*}$ & $0.71 \pm 0.10^{\dagger}$ & $0.65 \pm 0.08^{\dagger}$ & $1.34 \pm 0.12^{\ddagger}$ \\
\hline DT (ms) & $225.58 \pm 27.09^{*}$ & $238.53 \pm 11.41^{\dagger}$ & $240 \pm 10.43^{\dagger}$ & $193.17 \pm 12.29$ \\
\hline IVRT (ms) & $101.46 \pm 17.25^{\star}$ & $115.56 \pm 6.77^{\dagger}$ & $119.16 \pm 6.02^{\dagger}$ & $75.26 \pm 5.80^{\ddagger}$ \\
\hline
\end{tabular}

A, late velocity wave; DT, deceleration time; E, early velocity wave; $E^{\prime}$, early diastolic wave; EF, ejection fraction; IVRT, isovolumetric relaxation time; MPI, myocardial performance index; MV, mitral valve; PWD, pulsed-wave Doppler; S, systolic velocity wave; TDI, tissue Doppler imaging. ${ }^{*},,, \$$ statistically significant differences between groups with different symbols, but no significant differences between groups with the same symbol. 
Table 5 Comparison between compensated and decompensated patients in echocardiographic findings

\begin{tabular}{lccc}
\hline & \multicolumn{2}{c}{ Cases } & $P$ value \\
\cline { 2 - 3 } & Compensated & Decompensated & \\
\hline MPI PWD & $0.36-0.44$ & $0.38-0.62$ & \\
MPI TDI & $0.41 \pm 0.02$ & $0.44 \pm 0.05$ & 0.009 \\
& $0.41-0.51$ & $0.45-0.85$ & \\
EF by Simpson & $0.47 \pm 0.03$ & $0.52 \pm 0.09$ & 0.003 \\
& $56-71$ & $35-70$ & \\
Average S wave & $63.93 \pm 4.63$ & $60.1 \pm 8.05$ & 0.005 \\
& $9-14$ & $5-14$ & \\
EF by M mode & $12.33 \pm 1.35$ & $10.82 \pm 2.32$ & 0.001 \\
& $58-72$ & $33-70$ & \\
MV E/É ratio & $64.3 \pm 3.91$ & $60 \pm 8.46$ & 0.010 \\
& $4-19$ & $9-18$ & \\
MV E/A ratio & $13.33 \pm 4.45$ & $15.78 \pm 1.53$ & 0.000 \\
& $0.5-1.5$ & $0.5-1$ & \\
DT (ms) & $0.89 \pm 0.28$ & $0.69 \pm 0.1$ & 0.000 \\
& $168-288$ & $220-275$ & \\
IVRT (ms) & $225.87 \pm 27.09$ & $239.27 \pm 10.82$ & 0.000 \\
& $68-129$ & $96-135$ & \\
\hline A I & $101.47 \pm 17.25$ & $117.37 \pm 6.61$ & 0.000 \\
\hline
\end{tabular}

$A$, late velocity wave; $D T$, deceleration time; $E$, early velocity wave; $E^{\prime}$, early diastolic wave; EF, ejection fraction; IVRT, isovolumetric relaxation time; MPI, myocardial performance index; MV, mitral valve; PWD, pulsed wave Doppler; S, systolic velocity wave; TDI, tissue Doppler imaging. $P<0.05$ considered significant.

function in 45 patients with cirrhosis and found that 17 (38\%) patients had diastolic dysfunction. They also found that the presence of diastolic dysfunction was not associated with the severity of liver cirrhosis, but the severity of diastolic dysfunction was correlated with Child score. Merli et al. [10] diagnosed diastolic dysfunction in $64 \%$ of their studied population. Lengyel et al. [15] studied 96 patients with cirrhosis by TDI and found that approximately one-third of the patients had diastolic dysfunction.

Pagourelias et al. [16] studied 77 men with cirrhosis and 20 healthy controls and unexpectedly found that $\mathrm{EF}$ is significantly higher in patients with cirrhosis than controls, but this higher EF was not associated with changes in longitudinal LV deformation, and the time to the peak basal and apical rotation was significantly delayed in patients with cirrhosis than controls. They also found significantly impaired indices of diastolic function in patients than controls. Sampaio et al. [5] found that diastolic dysfunction is present in $44 \%$ of patients in their study, but they did not find significant differences between patients and controls in EF. Rimbas et al. [17] found diastolic dysfunction in $47.8 \%$ of patients with cirrhosis in their study.

All the previously mentioned studies and many other studies confirm the presence of LV dysfunction in patients with cirrhosis. The main mechanisms of cardiac dysfunction are not well understood. However, several factors are present in patients with cirrhosis which could contribute to cardiac dysfunction: the presence of high levels of norepinephrine and downregulation of $\beta 1$ and $\beta 2$ receptors, which control heart rate and contraction [18]; reduced adrenergic positive inotropic effect [19]; and reduced chronotropic cardiac response to stress [20]. Moreover, the overproduction of nitric oxide, the potent vasodilator which also inhibits $\beta$-adrenergic receptor stimulation and decreases contractility [21,22]. Carbon monoxide produced from degradation of heme also has a role in cardiac dysfunction in patients with cirrhosis [23], endogenous cannabinoids [24], and interference with the G-protein-coupled inhibitory receptors GB-1 are encountered factors that could contribute to cardiac dysfunction in patients with cirrhosis [25].

\section{Limitations}

Our study has many limitations: being cross-sectional, small sample size, and exclusion of patients with hyperdynamic circulation and those taking $\beta$-blockers, which represent a large fraction of patients with cirrhosis.

\section{Financial support and sponsorship}

Nil.

\section{Conflicts of interest}

There are no conflicts of interest.

\section{References}

1 Alqahtani SA, Fouad TR, Lee SS. Cirrhotic cardiomyopathy. Semin Liver Dis 2008; 28:59-69.

2 Moller S, Henriksen JH. Cardiovascular complications of cirrhosis. Gut 2008; 57:268-278.

3 Wong F. Cirrhotic cardiomyopathy. Hepatol Int 2009; 3:294-304.

4 Kazankov K, Holland-Fischer P, Andersen NH, Torp P, Sloth E, Aagaard NK, et al. Resting myocardial dysfunction in cirrhosis quantified by tissue Doppler imaging. Liver Int 2011; 31:534-540.

5 Sampaio F, Pimenta J, Bettencourt N, Fontes-Carvalho R, Silva AP, Valente J, et al. Systolic and diastolic dysfunction in cirrhosis: a tissueDoppler and speckle tracking echocardiography study. Liver Int 2013; 33:1158-1165.

6 Sampaio F, Pimenta J. Left ventricular function assessment in cirrhosis: current methods and future directions. World J Gastroenterol 2016; 22:112-125.

7 Valeriano V, Funaro S, Lionetti R, Riggio O, Pulcinelli G, Fiore P, et al. Modification of cardiac function in cirrhotic patients with and without ascites. Am J Gastroenterol 2000; 95:3200-3205.

8 Lang RM, Badano LP, Mor-Avi V, Afilalo J, Armstrong A, Ernande L, et al. Recommendations for cardiac chamber quantification by echocardiography in adults: an update from the American Society of Echocardiography and the European Association of Cardiovascular Imaging. Eur Heart J Cardiovasc Imaging 2015; 16:233-270.

9 Dorosz JL, Lezotte DC, Weitzenkamp DA, Allen LA, Salcedo EE. Performance of 3-dimensional echocardiography in measuring left ventricular volumes and ejection fraction: a systematic review and metaanalysis. J Am Coll Cardiol 2012; 59:1799-1808.

10 Merli M, Calicchia A, Ruffa A, Pellicori P, Riggio O, Giusto M, et al. Cardiac dysfunction in cirrhosis is not associated with the severity of liver disease. Eur J Intern Med 2013; 24:172-176. 
11 Nagueh SF, Appleton CP, Gillebert TC, Marino PN, Oh JK, Smiseth OA, et al. Recommendations for the evaluation of left ventricular diastolic function by echocardiography. J Am Soc Echocardiogr 2009; 22:107-133.

12 Kamath PS, Wiesner RH, Malinchoc M, Kremers W, Therneau TM, Kosberg CL, et al. A model to predict survival in patients with end-stage liver disease. Hepatology 2001; 33:464-470.

13 Hammami R, Boudabbous M, Jdidi J, Trabelsi F, Mroua F, Kallel R, et al. Cirrhotic cardiomyopathy: is there any correlation between the stage of cardiac impairment and the severity of liver disease? Libyan J Med 2017; 12:1283162.

14 Karagiannakis DS, Vlachogiannakos J, Anastasiadis G, Vafiadis-Zouboulis I, Ladas SD. Diastolic cardiac dysfunction is a predictor of dismal prognosis in patients with liver cirrhosis. Hepatol Int 2014; 8:588-594.

15 Lengyel M, Nagy A, Zorandi A. Tissue Doppler echocardiography: a new technique to assess diastolic function. Orv Hetil 2002; 143:333-339.

16 Pagourelias ED, Sotiriou P, Papadopoulos CE, Cholongitas E, Giouleme O, Vassilikos V. Left ventricular myocardial mechanics in cirrhosis: a speckle tracking echocardiographic study. Echocardiography 2016; 33:223-232.

17 Rimbas RC, Baldea SM, Guerra R, Visoiu SI, Rimbas M, Pop CS, et al. New definition criteria of myocardial dysfunction in patients with liver cirrhosis: a speckle tracking and tissue doppler imaging study. Ultrasound Med Biol 2018; 44: 562-574.
18 Lee SS, Marty J, Mantz J, Samain E, Braillon A, Lebrec D. Desensitization of myocardial beta-adrenergic receptors in cirrhotic rats. Hepatology 1990; 12(Pt 1):481-485.

19 Ma Z, Miyamoto A, Lee SS. Role of altered beta-adrenoceptor signal transduction in the pathogenesis of cirrhotic cardiomyopathy in rats. Gastroenterology 1996; 110:1191-1198.

20 Shujaat A, Bajwa AA. Optimization of preload in severe sepsis and septic shock. Crit Care Res Pract 2012; 2012:761051.

21 Milani A, Zaccaria R, Bombardieri G, Gasbarrini A, Pola P. Cirrhotic cardiomyopathy. Dig Liver Dis 2007; 39:507-515.

22 Garcia-Estan J, Ortiz MC, Lee SS. Nitric oxide and renal and cardiac dysfunction in cirrhosis. Clin Sci 2002; 102:213-222.

23 Suematsu $M$, Ishimura $Y$. The heme oxygenase-carbon monoxide system: a regulator of hepatobiliary function. Hepatology 2000; 31:3-6.

24 Ros J, Claria J, To-Figueras J, Planaguma A, Cejudo-Martin P, FernandezVaro $G$, et al. Endogenous cannabinoids: a new system involved in the homeostasis of arterial pressure in experimental cirrhosis in the rat. Gastroenterology 2002; 122:85-93.

25 Bonz A, Laser M, Kullmer S, Kniesch S, Babin-Ebell J, Popp V, et al. Cannabinoids acting on $\mathrm{CB} 1$ receptors decrease contractile performance in human atrial muscle. J Cardiovasc Pharmacol 2003; 41:657-664. 